\title{
Game Equilibrium of False Information Diffusion in Public Crisis on Interpersonal Network
}

\author{
Kai $\mathrm{LI}^{1, \mathrm{a}}$ and Jia-Jia $\mathrm{HAO}^{2}$ \\ ${ }^{1}$ Foreign Affairs Center of Ministry Industry and Information Technology Beijing, China \\ ${ }^{2}$ School of Economics and management Yanshan University Qinghuangdao, China
}

\begin{abstract}
The public crisis evolution will make a variety of impact on society, the public and the mass media will also focus on public crisis. In the evolution of public crisis, objective and non-objective information about the crisis will arise. Such harmful but not objective information is "false information". Various factors working together lead to the false information diffusion in public crisis. We will identify eight factors inducing false information in public crisis, and study the game equilibrium process of false information diffusion in public crisis on interpersonal network, and determine the equilibrium which was looked for. When the public identify that their interests are lower than the equilibrium, the false information is accepted, then false information on this node begins diffusing; When the public identify that their interests are higher than the equilibrium, the false information is identified, then diffusion on this node ends.
\end{abstract}

\section{Introduction}

With the rapid development of economic, the social and economic environment will become more complex and difficult to predict. By this time, the probability of public crisis occurring closely together will be enhanced greatly. When the public crisis happens, people tend to express their views of the event. But in the process of information diffusion, the content of the information may conflict with true information due to some artificial and inevitable factors. The false information such as rumor, gossip, hearsay, which is not in accordance with real information will surely contribute to the fear and anxiety of the public create more factors that lead to social instability. This is an main source of secondary damage of public crisis.

So what is the connotation of the "false information" in public crisis? What are the factors that led to the false information in public crisis? What kind of role did the inducing factors play in the spreading of false information in public crisis of game equilibrium? The research of these propositions has great importance in the scientific definition of the false information in public crisis. Meanwhile, to specify the inducing factors of false information in public crisis and their roles in the spread of game equilibrium is critical for us to grasp the development direction of the false information in public crisis and the factors that public care about in the event development .

\footnotetext{
${ }^{\text {a }}$ Corresponding author: likaigary@163.com
} 


\section{The connotation of the false information in public crisis}

The evolution of the public crisis is actually a series of diffusion effects of crisis information[1]. With the appearing of an huge amounts of information, some discordant voices will arise. It will raise the possibility of public who lack recognition to echo it. At this time "false information" will appear. What is "false information"? "False information" is not the information in a real sense, it's the information that is "untruthfulness or unrealistic"[2]. False information has some other appellations, such as dummy message, inferior information, expanding information, the selfish information[3]. False information in public crisis is the information that looks like the real but actually not[4]. False information is the information transmitted in the non-public media channels. It is a kind of harmful information that is not true, not objective, not conductive to social stability and easy to mislead the public[5].

In addition to the research on the definition of false information, some scholars have studied the process of false information diffusion. They suggest that the essence of false information diffusion in public crisis is a class of complex nonlinear evolution process[6]. Thompson et al. established the rumor model which is similar to the Daley-Kendall model. Considering the effect of the population's diversity in the rumor spreading, they found that people's activity is the most sensitive parameter that will influence the spread of rumors. They also found that increasing activity can control the size of the rumor spreading[7]. Kawachi developed the dynamics model in finite and infinite dimensional with considering varying accepting degrees of different populations, and then derived the rumor diffusion threshold[8]. Based on the influence of different contact for eventually diffusion. Seki et al. discussed the results through mathematical simulation[9]. Huo et al. studied the interactive model between rumors spreading and the government's emergency utility and drew the phase diagram of the system through data simulation. On the basis of these, they put forward strategies and policy recommendations about emergency management[10]. Zhong et al. divided the information into real information and false information and discussed the process of crisis information diffusion from the perspective of ecology. They built the model of social crisis information diffusion which is based on Lotka-Volterra model. Then carried out simulation and empirical analysis for social crisis information diffusion and found that the diffusion of crisis information is closely related to the diffusion coefficient and diffusion rate[11].

Some of the researchers think that crisis information only has two states: Absolute truth and totally untrue. However, in our real life there is some information which seems real but actually not. There is also some which seems far from objective truth, but is the real information in fact. The reason of information become seemingly far from objective truth is that the partial distortion of strictly true objective information leads to the qualitative change of a small part information. Its essence is real and less harmful to the society. We define information with characters above as virtual information in this paper. The information that seems objectively true is false information as a whole with a part of the true appearance. We defined the information as the false information in this paper. The purpose of this study is to reveal the game equilibrium of false information diffusion. As long as the information is related to the public crisis, whether rumors or gossip or rumors are included in this paper.

\section{Assumptions of false information Diffusion in public crisis}

The process of false information diffusion in public crisis is a process of decision-making in two sides. There are utility values for people to evaluate what decisions should be made, which means the value of maximum satisfaction. So the diffusion of false information in public crisis is a process of game equilibrium. But in real life, the public are restrained by attention, emotion, ability and personal psychological, so the decisions they made are limited. The decision-making process is not complete and accurate under the interference of each external environment, which means that it is an incomplete and imperfect decision-making process.

When the false information in public crisis generated, individuals try to express their views from the aspects of the false information inducing factors in public crisis and to persuade other individuals 
to judge the fairness and security of the environment from these perspectives as well. Therefore, the process of false information diffusion in public crisis is the result from interactions of the eight factors which are mentioned above. Next, we will study the game equilibrium of false information diffusion in public crisis from the eight aspects.

Assumption1: Information of different aspects which the recipients try to express is independent, which means that the eight factors: culture, personal life needs, interpersonal relationship, group attitude, profit distribution, the sanity of legal system, the government credibility and development coordination are not related to each other. One of the intentions that contained in the comments is represented by $i$, the total information of type $i$ is represented by $I_{i}=x_{i}+\varepsilon_{i}$. Where $I_{i}$ represent the amounts of $i, \varepsilon_{i}$ is the amount of uncertainty of the intention. $\varepsilon_{i}$ is one of the normal distribution random variables. Then, we have $E\left(\varepsilon_{i}\right)=0, \operatorname{var}\left(\varepsilon_{i}\right)=\delta_{i}^{2}$. The total intention of a particular aspect that individuals express is: $I=\sum x_{i}+\sum \varepsilon_{i}$.

Hypothesis2: The individuals who haven't accept the false information in public crisis will identify the information of their surrounding environment in all aspects which are independent. It means that they can judge fairness and security from the eight factors of culture, personal life needs, interpersonal relationship, group attitude, profit distribution, the sanity of legal system, the government credibility, and development coordination are not related to each other. The intention of a particular aspect distinguished in the comments of the individual who accepted false information can be represented by $i$ in public crisis. The total information of type $i$ is represented by $D_{i}=x_{i}+\varepsilon_{d i} . D_{i}$ represent the amounts of $i \cdot \varepsilon_{d i}$ is a normal distribution random variable which represents the amount of uncertainty intention. We can derive the formula: $E\left(\varepsilon_{d i}\right)=0, \operatorname{var}\left(\varepsilon_{d i}\right)=\delta_{d i}^{2}$. The relevance between $\varepsilon_{i}$ and $\varepsilon_{d i}$ is $\rho_{i}$. spreaders' intention from eight aspects are represented by $i$. The total intention of a particular aspect that individuals express is $D=\sum x_{i}+\sum \varepsilon_{d i}$.

Hypothesis3: When the false information recipient express their intentions, the more information they express the more satisfied they are. In this article, we assume that this kind of satisfaction is $\pi$. It is proportional to the total amount of information they express. When the individuals who haven't accept false information distinguish the information, the more information they identify, the more satisfied they are. In this article, we assume that this kind of satisfaction is $P$, which is proportional to the total information that they identify. For the sake of simplicity, we assume:

$$
\begin{gathered}
\pi=\sum \pi_{i}=I=\sum I_{i}=\sum x_{i}+\sum \varepsilon_{i} \\
P=\sum P_{i}=D=\sum D_{i}=\sum x_{i}+\sum \varepsilon_{d i}
\end{gathered}
$$

In the formula (1).(2), $i$ represents the eight kinds of intention contained in the false information in public crisis.

Hypothesis4: The utility of individual who haven't accepted the false information is the sense of achievement after judging the fairness of their surroundings and security. We set it as $\left.u\left(w, x_{i}\right)=e^{r(w}\left(x_{i}\right)\right)$. When the recipient expresses false information, the utility of is the satisfaction after resonate with other individuals. We set it as: $u\left(t_{i}, f_{i}, s_{i}\right)=E(\pi w)$. Where $\left(x_{i}\right)=\frac{1}{2} \sum c_{i} x_{i}^{2}$ is the emotional cost that the individuals who haven't accepted the false information spend on distinguishing the environment. $w=\sum w_{i}$ represents the satisfaction that the individuals who won't accept the false information have after identifying the false information(It also can be called the emotional cost that false information recipients paid ). It is the linear combination of $i$,

$w_{i}=t_{i}+f_{i} \pi_{i}+s_{i} p_{i} \cdot t_{i}$ represents the identity amount of the existing fixed environmental fairness and security. $f_{i}$ represents the rate of being persuaded, $s_{i}$ represents the rate of self- 
persuasion. $\pi w$ represents the false information recipients' sense of accomplishment after they persuaded others. $i$ represents the eight aspects of the intention that the individuals want to express.

Hypothesis5: The model of false information diffusion in public crisis assume that the recipients of false information in public crisis want to achieve their own maximization of satisfaction by spreading false information. Meanwhile the individual who does not accept false information in public crisis will not spread the false information in public crisis and make themselves the most satisfaction in participation utility condition.

\section{The equilibrium of the model}

The decision model that the recipients in public crisis spread false information and individuals who haven't accepted false information in public crisis identify the information is following:

$$
\begin{aligned}
& \max _{t_{i}, f_{i}, s_{i}} u\left(t_{i}, f_{i}, s_{i}\right)=E\left(\begin{array}{ll}
\pi & w
\end{array}\right) \\
& \text { S.t } \\
& \begin{array}{l}
x_{i} \in \underset{r(w)}{\arg \max } E\left[e^{r(w}\left(x_{i}\right)\right) \\
E\left[e^{r(w)}\right] \geqslant E\left[e^{r w_{0}}\right]
\end{array}
\end{aligned}
$$

Where, $w_{0}$ represents the satisfaction before they identify the false information.

The process of false information diffusion in public crisis is a incomplete process. Which has a variety of combinations. To solve the balance of many combinations is very complicated and unnecessary. While the complete false information diffusion model in public crisis are comprehensive and representative, we solve the equilibrium in the process of the complete diffusion in this paper.

A complete diffusion process of the model for certainty equivalent equation is following:

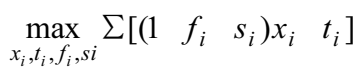

$$
\begin{aligned}
& \text { S.t } \left.\left.x \in \underset{x_{i}}{\arg \max _{i}\left\{\Sigma \left[\left(f_{i}+s_{i}\right) x_{i}\right.\right.} t_{i} \frac{c_{i} x_{i}^{2}}{2}\right] \frac{1}{2} \sum\left(f_{i}^{2} \delta_{i}^{2}+2 \rho_{i} s_{i} f_{d i}+s_{i}^{2} \delta_{d i}^{2}\right)\right\} \\
& \Sigma\left[\left(f_{i}+s_{i}\right) x_{i} t_{i} \frac{c_{i} x_{i}^{2}}{2}\right] \frac{r}{2} \sum\left(f_{i}^{2} \delta_{i}^{2}+2 \rho_{i} s_{i} f \delta_{d i}+s_{i}^{2} \delta_{d i}^{2}\right) \geqslant w_{0}
\end{aligned}
$$

The equilibrium of formula (4) is following:

$$
\begin{aligned}
f_{i}^{*} & =\frac{\delta_{i}^{2} \rho_{i} \delta_{i} \delta_{d i}}{\delta_{i}^{2} 2 \rho_{i} \delta_{i} \delta_{d i}+\delta_{d i}^{2}} \frac{1}{1++r c_{i} \Omega_{i}} \\
s_{i}^{*} & =\frac{\delta_{d i}^{2} \rho_{i} \delta_{i} \delta_{d i}}{\delta_{i}^{2} 2 \rho_{i} \delta_{i} \delta_{d i}+\delta_{d i}^{2}} \frac{1}{1++r c_{i} \Omega_{i}} \\
x_{i}^{*} & =\frac{f_{i}^{*}+s_{i}^{*}}{c_{i}}=\frac{1}{c_{i}\left(1++r c_{i} \Omega_{i}\right)} \\
I^{*} & =D^{*}=\sum x_{i}^{*}=\sum \frac{1}{c_{i}\left(1++r c_{i} \Omega_{i}\right)} \\
w^{*} & =\sum w_{i}^{*}=\sum\left[t_{i}+\frac{1}{c_{i}\left(1++r c_{i} \Omega_{i}\right)^{2}}\right]
\end{aligned}
$$

In the formula of (5).(6).(7).(8).(9), $\Omega_{i}=\frac{\delta_{i}^{2} \delta_{d i}^{2}\left(1 \rho_{i}\right)}{\delta_{i}^{2}+\delta_{d i}^{2} 2 \rho_{i} \delta_{i} \delta_{d i}}, i$ represents eight kinds of intention that contained in false information in public crisis. 


\section{The game equilibrium rule of false information diffusion in public crisis on interpersonal network}

(1) The equilibrium of false information diffusion in public crisis is determined by the following factors:

When the recipients of false information in public crisis spread information, the attitude stability is $\delta_{i}^{2}$. The certainty of individuals who haven't accepted false information for fairness and security of the environment they lived is $\delta_{d i}^{2}$;

The fairness and safety factor that the individuals who haven't accepted false information in public crisis avoid the misjudgment for environment is $r$;

The emotional cost that individuals who haven't accepted false information in public crisis will pay to identify the environmental fairness and security is $c_{i}$;

The relevance between recipients spreading information and individuals who haven't accepted the false information identifying it is represented by $\rho_{i}$.

Through the analysis above, we can know that the process of false information diffusion in public crisis is the communication between the spreader and the which is a result of interaction between subjective and objective factors.

The objective factors refer to the fairness and security of realistic environment. The subjective factors refer to the tendency that both of them express and identify information, psychological reactions and avoiding miscalculation. These factors will affect the transmission and the recognition of information, and make fluctuations in information diffusion process, which form the main reason for information difficult to control.

(2) The diffusion process of false information in public crisis is the process that the public judge the nature of the environment from multiple aspects and make a decision. The public is emotional complex, so the decision-making process is also the outcome of various complicated factors interaction. According to the analysis on the result of game equilibrium, the game equilibrium of false information diffusion process in public crisis is that intentions, the rate of being persuaded, the rate of self-persuasion change according to deciding factors' changes. The differences of marginal changes are shown in Table 1.

Table 1. The game equilibrium of false information diffusion in public crisis varies with each factor

\begin{tabular}{|c|c|c|}
\hline Factors & $f_{i}^{*}$ & $s_{i}^{*}$ \\
\hline$\delta_{i}$ & $\frac{\partial M}{\partial \delta_{i}} \frac{1}{G} \frac{r c_{i} M}{G^{2}} \frac{\partial \Omega_{i}}{\partial \delta_{i}}$ & $\frac{\partial N}{\partial \delta_{i}} \frac{1}{G} \frac{r c_{i} N}{G^{2}} \frac{\partial \Omega_{i}}{\partial \delta_{i}}$ \\
\hline$\delta_{d i}$ & $\frac{\partial M}{\partial \delta_{d i}} \frac{1}{G} \frac{r c_{i} M}{G^{2}} \frac{\partial \Omega_{i}}{\partial \delta_{d i}}$ & $\frac{\partial N}{\partial \delta_{d i}} \frac{1}{G} \frac{r c_{i} N}{G^{2}} \frac{\partial \Omega_{i}}{\partial \delta_{d i}}$ \\
\hline$c_{i}$ & $\frac{r c_{i} M}{G^{2}} \frac{\partial \Omega_{i}}{c_{i}}$ & $\frac{r c_{i} N}{G^{2}} \frac{\partial \Omega_{i}}{c_{i}}$ \\
\hline$\rho_{i}$ & $\frac{\partial M}{G} \frac{r c_{i} M}{G^{2}} \frac{\partial \Omega_{i}}{\rho_{i}}$ & $\frac{r c_{i} N}{G} \frac{\partial \Omega_{i}}{\sigma_{i}}$ \\
\hline$r$ & $\frac{c_{i} M \Omega_{i}}{G^{2}}$ & $\frac{c_{i} N \Omega_{i}}{G^{2}}$ \\
\hline
\end{tabular}


In Table $1, M=\frac{\delta_{i}^{2} \rho_{i} \delta_{i} \delta_{d i}}{\delta_{i}^{2} 2 \rho_{i} \delta_{i} \delta_{d i}+\delta_{d i}^{2}}$

$$
\begin{aligned}
& N=\frac{\delta_{d i}{ }^{2} \rho_{i} \delta_{i} \delta_{d i}}{\delta_{i}^{2} 2 \rho_{i} \delta_{i} \delta_{d i}+\delta_{d i}{ }^{2}}, \\
& G=1++r c_{i} \Omega_{i}, \\
& \Omega_{i}=\frac{\delta_{i}^{2} \delta_{d i}^{2}\left(1 \quad \rho_{i}\right)}{\delta_{i}^{2}+\delta_{d i}^{2} 2 \rho_{i} \delta_{i} \delta_{d i}}
\end{aligned}
$$

The higher $\delta_{i}^{2}$ (the attitude stability of false information recipient in public crisis when express information) is, the higher the rate of self-persuasion is. At the same time, the possibility of the individuals who haven't accepted the false information in public crisis being persuaded is higher; The higher $\delta_{d i}^{2}$ (the certainty the individuals have in environment fairness and security)is, the lower the rate of being persuaded and the rate of self-persuasion are.

The higher $r$ (The factors that the individual who haven't accepted false information in public crisis) avoid the misjudgment for the environmental fairness and safety is, the lower the possibility of being persuaded and self-persuasion are. The more clearly that public can understand the environment around, the less willingly they want to waste time and effort to identify environmental justice and security. The result is that the probability to accept false information in public crisis is low.

When $\rho=0$, the behavior of the individuals who spread the false information in public crisis is not related to the intention of the individuals who haven't accepted the false information in public crisis. When the false information recipients in public crisis express the false information, the uncertainty of expression will decline the probability of being persuaded. Meanwhile the ability of them to identify false information in public crisis will decrease and the satisfaction after identification will also fall. At this time, this information will only be accepted by a few people. The reason for this phenomenon is that the information is strictly false information and has small correlations with other individuals. It shows that the false information that can be diffused is a kind of information which is related to people's satisfaction in many aspects, which means the false information in public crisis that can be diffused is the information that is related to people's vital interests and life desires. This is in accordance with the definition in this paper.

When $c_{i}$ (the emotional cost that individual who haven't accepted false information in public crisis will pay to identify the environmental fairness and security.)increases, the rate of being persuaded and the rate of self-persuasion will fall.

(3) When the spreaders spread false information or the individuals who haven't accepted the information recognize it, the most fundamental motivating factors are different. It means that the reasons that different individuals accept or refuse false information are different. It's not single factor, but the interaction of various objective and subjective factors. The false information spreader will enrich the content of false information according to their own circumstances. At the same time, the individuals will identify the false information according to the information provided by the spreaders and their own conditions, which will add to the complexity in game process of spreading and recognizing false information.

(4) The satisfaction after recognizing false information changes according to the fluctuation of certainty for each inducing factors in all aspects. It means that the certainty attitude of environmental fairness and security that the individual who haven't accepted the false information in public crisis is associated with the satisfaction after they identify it. The emotion cost that the recipient in public crisis paid is related to the attitude stability of environmental fairness and security.

(5) When $\frac{\delta_{d i}{ }^{2} \rho_{i} \delta_{i} \delta_{d i}}{\delta_{i}^{2} 2 \rho_{i} \delta_{i} \delta_{d i}+\delta_{d i}{ }^{2}} \frac{1}{1++r c_{i} \Omega_{i}}>\frac{\delta_{d j}{ }^{2} \rho_{i} \delta_{j} \delta_{d j}}{\delta_{i}^{2} 2 \rho_{i} \delta_{j} \delta_{d j}+\delta_{d j}{ }^{2}} \frac{1}{1++r c_{j} \Omega_{j}}$, the rate of selfpersuasion though intention $i$ is higher than the intention $j$ for the individuals who haven't identified 
the false information in public crisis, $s_{i}^{*}>s_{j}^{*}$. It means that we can identify the false information in public crisis easier according to the intention of $i$.

$$
\text { whe } \frac{\delta_{i}^{2} \rho_{i} \delta_{i} \delta_{d i}}{\delta_{i}^{2} 2 \rho_{i} \delta_{i} \delta_{d i}+\delta_{d i}^{2}} \frac{1}{1++r c_{i} \Omega_{i}}>\frac{\delta_{j}^{2} \rho_{i} \delta_{j} \delta_{d j}}{\delta_{i}^{2} 2 \rho_{i} \delta_{j} \delta_{d j}+\delta_{d j}{ }^{2}} \frac{1}{1++r c_{j} \Omega_{j}} \text {, the rate of being persuaded }
$$
through intention $i$ is higher than the intention $j$ for the individual who haven't accepted the false information in public crisis, $f_{i}^{*}>f_{j}^{*}$. It means that the spreaders and individuals who haven't accepted information resonate on the intention $i$. In this case, the individuals who haven't accepted the false information in public crisis will accept false information easily. The individuals who haven't accepted the false information in public crisis should distinguish the intention selectively. Sometimes they cannot obtain the greatest satisfaction even exhausted all intentions. It can also lead to excessive information identification and increase dissatisfaction.

\section{Diffusion process of false information in public crisis on interpersonal network}

The thought of liberty and democracy spur people to continually judge the fairness of the surroundings, especially when public crisis happens. With the increasing frequency of judgment, the of social development and the imbalance of the profit distribution lead to dissatisfaction with their surroundings. At this time, people want to express their dissatisfaction by proper channels and they expect the dissatisfaction can be solved. But some evasive and laissez faire behaviors of some departments have intensified people's sense of injustice. So people will express their views in the range of their communication, which can't show the real facts completely. The information with part of distortion mingles with the citizen's personal feelings of events and it is biased. But the unfairness and security of their surrounding environment let them believe that their views of the event are correct. Thus the false information in public crisis will be inspired. The process is shown in Figure 1.

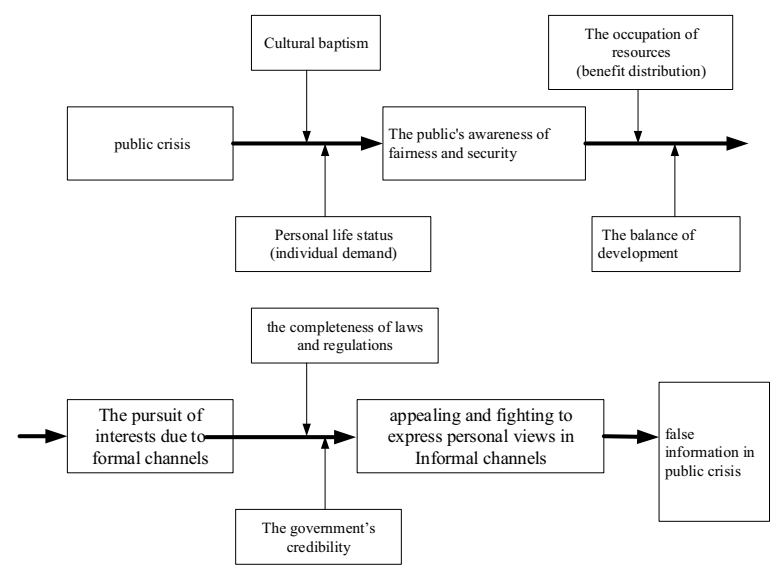

Figure 1. The emerging process of false information in public crisis

When false information in public crisis generates, the public will judge the fairness according to the resources they occupied, the culture they accepted, their personal status, etc. If the public's decision results are that the public environment is fair, then the false information in public crisis does not involve the public interest. The enthusiasms of the public to express false information will disappear. This is the phenomenon that "we don't care the thing that has nothing to do with us". They just take the event or situation as the topic of communication rather than take it as a way of expressing 
social discontent. So the false information in public crisis is identified successfully and will stop spreading. And when the public's decision results are that they are not treated fairly or they feel the environment around them isn't safe, they want to express their dissatisfaction in some ways. If they get the deserved interests from normal complaint channels, it will stop spreading. If the complaint through formal channels is not successful, people will choose to accept false information and diffuse it in their interpersonal network. The process is shown in Figure 2.

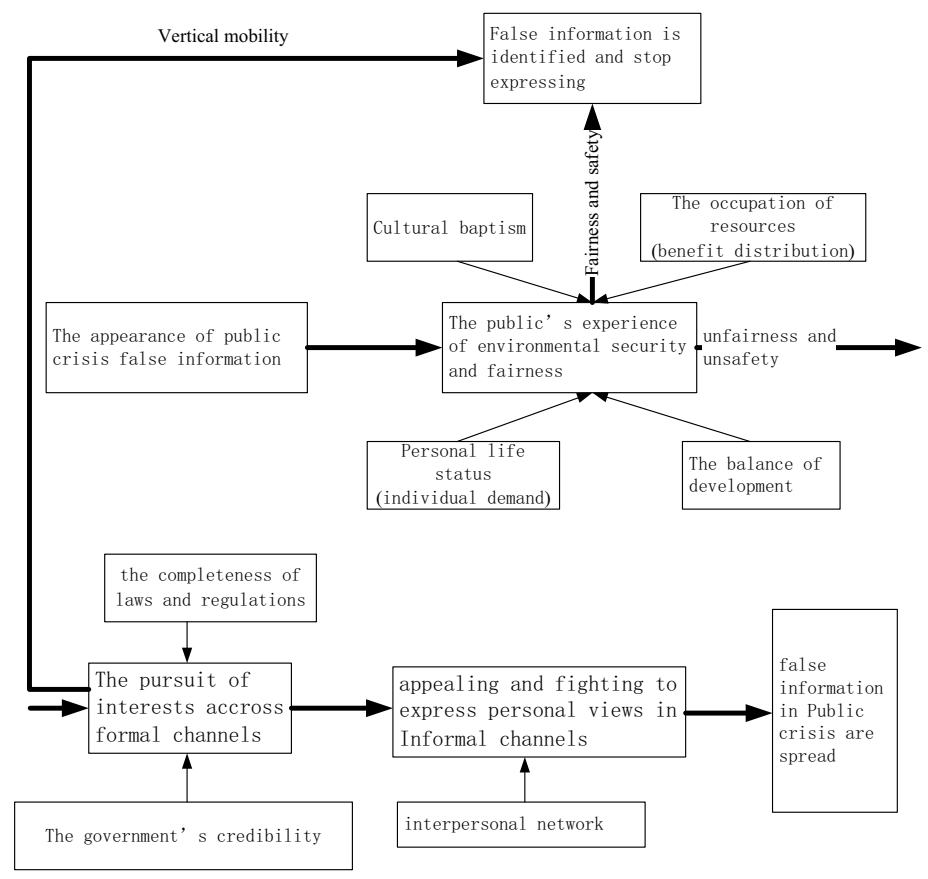

Figure 2.The spread process of false information in Public crisis

\section{The interventions of false information diffusion in public crisis}

(1) When public crisis generates, some objective and subjective factors, which contain the fairness and safety of the real environment, the tendency of different individual to spread and identify the false information, psychological reaction, avoiding mistakes, will affect the process of false information diffusion. They will lead to false information spreading or stopping spreading. At the same time, the interaction of subjective and objective factors increase supervision difficulties of false information in public crisis. When we want to intervene the process of false information diffusion in public crisis, we can begin from two aspects.

From the aspects of the external soft environment, the relevant departments should plan as a whole, increase the balance of social development, reduce the phenomenon of distribution unequally and increase public's satisfaction of their surroundings. Beyond that, the relevant departments should also increase the degree of supervision and regulation from the environment for living. For example, they can meet the public's needs of safe environment from the aspect of environmental enhancement, improving medical level, improving the quality of education, raising employment rate, etc. They should complete the corresponding laws and regulations, and specify the responsibility scope of the relevant departments. When the public interests are violated and complain to the relevant departments, 
the departments should resolved it in time. The phenomenon of shirking responsibility and omission should be put an end in order to increase the public's sense of security.

From the aspects of the public themselves, the stability of attitude, the emotion cost of identifying false information in public crisis and the part authenticity of the false information's kernel are the factors that can affect individuals to accept false information. That is the instability of external environment can make the public to suspect their safety. The relevant departments should establish feasible crisis guarantee system and implement it in order to reduce the doubts of the public. They should do a good job of crisis aftermath, eliminate the motion of unfair and insecurity, enhance the public's certainty degree of environmental fairness and safety, which means they should reduce the cost of identification of the false information and the attitude stability. Thus when public crisis happens, the public are not willing to and also have no need to spread false information.

(2)When the spreaders spread false information or the individuals who haven't accepted the information recognize it, the most fundamental motivating factors are different. It means that the reasons that different individuals accept or refuse false information are different. The individuals will distinguish the intention selectively. Sometimes they cannot obtain the greatest satisfaction even exhausted all intentions. It can also lead to excessive information identification and increase dissatisfaction. If they resonate on intentions, the individuals will accept the crisis information. So when the false information generates, the relevant departments should intervene it according to different principal contradictions in different areas, which requires the apartments to do well in crisis management and specify the principal contradictions that will dissatisfy the public in different areas.

(3) False information in public crisis is concerned more by the public. If force to stop its spreading, we will infuriate the spreaders and make matters worse. Indirect measures should be taken in the relevant departments, rather than deny part of the authenticity .At the same time in the process of resource allocation, we should insist on human-oriented principle, the principle of high efficiency, sustainability, fairness principle and reduce the degree of public's complaint and dissatisfaction about the social system and decrease the number of inducing factors about false information diffusion in public crisis

\section{References}

1. Z. Gang, X. Q. Yang, Info. J, 22-25 (2006)

2. X. Feng, tech. Phil, People's Publishing House, (2007)

3. W. d. Cheng, Jinggangshan University. J, (Social Sciences),54-59( 2012)

4. L. Tuo, Journal of Information, 155-157 (2008)

5. L. Tuo, F. W. Yu, Z.g. Fa, Info. Stud:Theo\& Appl, 825-828 (2008)

6. F. W. Yu, L. Tuo, Z. g. Fa, Journal of Information, 8(2007)

7. K. Thompson, Tech. Repo BU-1642-M,( 2003)

8. K. Kawachi, Nonlinear Analysis: Real World Applications , 1989 -2028( 2008)

9. K. Kawachi, M. Seki, Theo Biol. J, 253, 55-60(2008)

10. L. A. Huo, P. Q. Huang, X .Fang, Phys A: Stat. Mech. Appl, 1, 3267-3274(2011)

11. Q. Zhong, W. Qi, L. Zhang. Syst. Engi-Theo\&Prac, 32, 104-110(2012) 